\title{
Clinicopathological Characteristics of Squamous Cell Carcinoma and High-grade Squamous Intraepithelial Lesions Involving Endocervical Polyps
}

\author{
CHEOL KEUN PARK ${ }^{1 *}$, YONG-WOOK KIM ${ }^{2 *}$, HYUN HEE KOH ${ }^{3}$, \\ NARA YOON ${ }^{4}$, GO EUN BAE ${ }^{5}$ and HYUN-SOO KIM ${ }^{3}$ \\ ${ }^{1}$ Department of Pathology, Severance Hospital, Yonsei University College of Medicine, Seoul, Republic of Korea; \\ ${ }^{2}$ Department of Obstetrics and Gynecology, Incheon St. Mary's Hospital, College of Medicine, \\ The Catholic University of Korea, Incheon, Republic of Korea; \\ ${ }^{3}$ Department of Pathology and Translational Genomics, Samsung Medical Center, \\ Sungkyunkwan University School of Medicine, Seoul, Republic of Korea; \\ ${ }^{4}$ Department of Pathology, Incheon St. Mary's Hospital, College of Medicine, \\ The Catholic University of Korea, Incheon, Republic of Korea; \\ ${ }^{5}$ Department of Pathology, Chungnam National University Hospital, \\ Chungnam National University School of Medicine, Daejeon, Republic of Korea
}

\begin{abstract}
Background/Aim: To investigate the clinicopathological characteristics of high-grade squamous intraepithelial lesions (HSILs) and squamous cell carcinomas (SCCS) involving endocervical polyps (ECPS). Patients and Methods: We collected the endocervical polypectomy cases and performed pathological examination and cytohistological correlation. Results: During a period of 12 years, 21 (1.1\%) HSILs and two (0.1\%) SCCs involving ECPs were identified in 1,905 cases. Twelve (63.1\%) of the 19 cases were cytohistologically concordant. In five HSILs and one SCC with polypectomy margin involvement, residual HSIL was identified in conization or hysterectomy specimens. Furthermore, in two HSIL patients and one SCC patient with
\end{abstract}

This article is freely accessible online.

*These Authors contributed equally to this study.

Correspondence to: Hyun-Soo Kim, Department of Pathology and Translational Genomics, Samsung Medical Center, Sungkyunkwan University School of Medicine, 81, Irwon-ro, Gangnam-gu, Seoul 06351, Republic of Korea. Tel: +82 234101243, Fax: +82 234132831, e-mail: hyun-soo.kim@samsung.com; Go Eun Bae, Department of Pathology, Chungnam National University Hospital, Chungnam National University School of Medicine, 282 Munhwaro, Jung-gu, Daejeon 35015, Republic of Korea. Tel: +82 422807797, Fax: +82 422807189, e-mail: goeunbae1@gmail.com

Key Words: Cervix, endocervical polyp, squamous cell carcinoma, high-grade squamous intraepithelial lesion. negative polypectomy margins, residual HSILs were found in the conization specimens. Conclusion: The prevalence of HSIL and SCC involving ECP in our cohort was similar to the rates found in previous studies. The presence of residual HSIL in nonpolypoid cervical tissue regardless of the polypectomy margin involvement suggests that conization or hysterectomy is needed for diagnostic or treatment purposes.

Endocervical polyps (ECPs) are the most common benign neoplasms of the uterine cervix that characteristically arise in the endocervical canal (1). They can occur over a wide age range, but about $90 \%$ of patients are aged $>40$ years (2). The reported prevalence of ECP is $1.5-10 \%$, and its pathogenesis remains unknown. Focal reactive epithelial and stromal hyperplasia associated with repeated episodes of inflammation, an abnormal local response to increased estrogen levels, or local congestion of cervical stromal blood vessels might be involved in the development of $\operatorname{ECP}(1,3)$. Approximately $60 \%$ of ECP patients is asymptomatic and discovered incidentally during routine gynecologic examinations, whereas symptomatic ECPs manifest as menorrhagia, metrorrhagia, postcoital bleeding, postmenopausal bleeding, vaginal discharge, or leukorrhea $(2,4)$.

Even though the reported prevalence of malignancies arising from ECP is $0.1-0.5 \%$, a thorough literature review revealed no information about malignancies involving ECP in Asian women $(2,3)$. We recently experienced several patients with high-grade squamous intraepithelial lesions (HSIL) and squamous cell carcinoma (SCC) involving ECP, which initiated a comprehensive review of our archival 
Table I. Clinical characteristics of high-grade squamous intraepithelial lesion (HSIL) and squamous cell carcinoma (SCC) involving endocervical polyp.

\begin{tabular}{|c|c|c|c|c|}
\hline \multirow[t]{2}{*}{ Characteristic } & & \multicolumn{2}{|c|}{ Polypectomy diagnosis } & \multirow[t]{2}{*}{ Total } \\
\hline & & HSIL & SCC & \\
\hline \multicolumn{2}{|l|}{ Number of cases } & 21 & 2 & 23 \\
\hline Age (years) & Mean (range) & $49.9(30-84)$ & $58(53-63)$ & $50.6(30-84)$ \\
\hline \multirow[t]{3}{*}{ Human papillomavirus status } & High-risk detected & 15 & 1 & 16 \\
\hline & Not detected & 2 & 0 & 2 \\
\hline & Not applicable & 4 & 1 & 5 \\
\hline \multirow[t]{6}{*}{ Cytology diagnosis } & $\mathrm{SCC}$ & 0 & 1 & 1 \\
\hline & HSIL & 5 & 0 & 5 \\
\hline & Atypical squamous cells, cannot exclude HSIL & 6 & 0 & 6 \\
\hline & Low-grade squamous intraepithelial lesion & 1 & 0 & 1 \\
\hline & Atypical squamous cells of undetermined significance & 4 & 1 & 5 \\
\hline & Negative for intraepithelial lesion or malignancy & 1 & 0 & 1 \\
\hline \multirow{4}{*}{$\begin{array}{l}\text { Surgical treatment } \\
\text { after polypectomy }\end{array}$} & Conization only & 6 & 1 & 7 \\
\hline & Hysterectomy only & 4 & 1 & 5 \\
\hline & Conization and hysterectomy & 3 & 0 & 3 \\
\hline & None & 8 & 0 & 8 \\
\hline Follow-up period & Mean (range) & $44.3(7-173)$ & $16(11-21)$ & $41.4(7-173)$ \\
\hline \multirow[t]{2}{*}{ Current status } & No evidence of disease & 17 & 2 & 19 \\
\hline & Not applicable & 4 & 0 & 4 \\
\hline
\end{tabular}

records. In this study, we aimed to determine the prevalence of HSILs and SCCs involving ECP and to investigate their clinical characteristics and pathological features, as well as the relationship between cytology and human papillomavirus (HPV) results.

\section{Patients and Methods}

Patient selection. This retrospective study (4-2016-0549) was reviewed and approved by the Institutional Review Board at Severance Hospital (Republic of Korea). Cases were collected by searching the pathology database for ECP between 1996 and 2018. The search included all reports with the terms "cervical", "endocervical", "polypoid", and "polyp". We identified 1,976 patients with ECP. Multiple polyps in an individual single patient discovered less than six months apart were taken as being the same polyp. When data from an individual patient remained unchanged for six months, only the most recent data were collected. Consequently, 71 cases were excluded. Among 1,905 cases of ECP, we extracted cases with HSIL or SCC involving ECP using the histological criteria recommended by the World Health Organization (WHO) Classification of Tumours of Female Reproductive Organs (5) and the Lower Anogenital Squamous Terminology (LAST) Standardization Project (6). The clinical features were determined from reviews of the electronic medical records, or by communicating with referring gynecologists. Images were reviewed where available. The primary lesions of all 1,905 patients were histologically assessed by two board-certified pathologists specialized in gynecological oncology. They examined all available hematoxylin and eosin-stained slides, concluded pathological diagnoses, and selected representative slides for immunostaining and HPV genotyping.

Immunohistochemical staining and interpretation. Sections $(4-\mu \mathrm{m}$ thick) cut from formalin-fixed, paraffin-embedded tissue blocks were placed onto Superfrost Plus glass slides (Thermo Fisher Scientific, Waltham, MA, USA). Thereafter, the sections were deparaffinized in xylene, rehydrated through a series of graded alcohols and immunohistochemically stained using an automated Ventana Benchmark XT (Ventana Medical Systems Inc.) as described by the manufacturer (7-13). Antigen was retrieved using CC1 Cell Conditioning Solution (Ventana Medical Systems Inc.). The sections were incubated with primary antibody for p16 using prediluted clone E6H4 (Ventana Medical Systems Inc.). After chromogenic visualization using an ultraView Universal DAB Detection Kit (Ventana Medical Systems Inc.), the slices were counterstained with hematoxylin, dehydrated in graded alcohols and xylene, and then embedded in mounting solution. Appropriate positive and negative controls were concurrently stained to validate the staining method. Cervical invasive squamous cell carcinoma and endometrial serous carcinoma tissue samples obtained by radical hysterectomy were used as positive controls. According to the recommendation of the LAST Standardization Project (6), the immunostaining profile of p16 was interpreted as block positive when 16 expression was horizontally continuous and strong, with nuclear or nuclear plus cytoplasmic staining. All other p16 immunostaining profiles, described as focal nuclear, wispy, bloblike, puddled, or scattered cytoplasmic staining were interpreted as patchy positive $(9,14-19)$. 

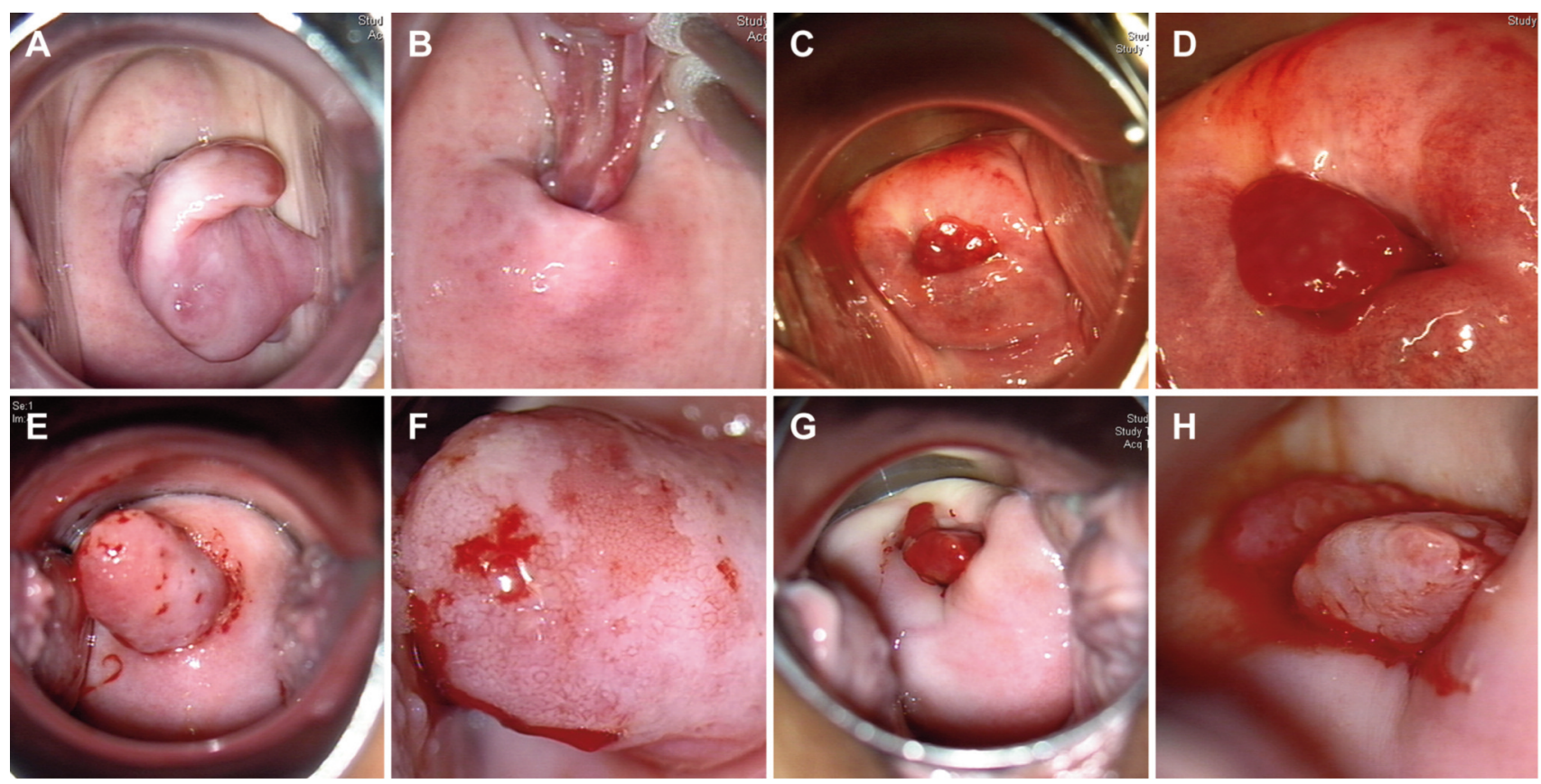

Figure 1. Colposcopy features of endocervical polyps. (A and B) Case 10. (A) Head of pedunculated polyp projects beyond external os. (B) Long stalk is apparently attached to endocervix. (C and D) Case 7. (C) Polyp protrudes from external os. (D) Small, deep red polyp resembles early granulation tissue formation. $(E$ and $F$ ) Case $11 .(E)$ Cylindrical polypoid mass protrudes from endocervical canal and widens external os. ( $F)$ Polyp surface has irregular-shaped superficial erosions and patchy hemorrhagic spots. ( $G$ and $H$ ) Case 13 . (G) Small polyp has similar shape to that in case 7. (H) Close-up view reveals gray-to-pink, slightly nodular appearance at surface.

HPV genotyping assay. We used a commercially available polymerase chain reaction (PCR)-based microarray with a HPV 9G DNA chip (BMT HPV 9G DNA Chip; Biometrix Technology) (2022). The $9 \mathrm{G}$ test detects 14 high-risk $(16,18,31,33,35,39,45,51$, $52,56,58,59,66$, and 68$)$ and five low-risk $(6,11,34,40$, and 42) HPV types as described by the manufacturer. Briefly, the PCR mixture contained $10 \mu \mathrm{L}$ of extracted target DNA, $10 \mu \mathrm{l}$ of BMT Primer set (Biometrix Technology), and PCR premix (Biometrix Technology) contained dNTP and Taq DNA polymerase in amplification buffer. Fragments of DNA were amplified using the following cycling program: pre-denaturation at $94^{\circ} \mathrm{C}$ for $5 \mathrm{~min}, 40$ denaturation cycles at $94^{\circ} \mathrm{C}$ for $30 \mathrm{~s}, 40$ annealing cycles at $45^{\circ} \mathrm{C}$ for $30 \mathrm{~s}, 40$ elongation cycles at $72^{\circ} \mathrm{C}$ for $30 \mathrm{~s}$, and final elongation at $72^{\circ} \mathrm{C}$ for $5 \mathrm{~min}$. The PCR products were resolved by electrophoresis in $2 \%$ agarose gels to confirm amplification. Each hybridization chamber of the $9 \mathrm{G}$ was covered with a mixture of the hybridization solution $(35 \mu \mathrm{l})$ and PCR products $(15 \mu \mathrm{l})$ and incubated at $23^{\circ} \mathrm{C}-26^{\circ} \mathrm{C}$ for $30 \mathrm{~min}$. The array was washed, then images were acquired using a fluorescent ScanArray GX Microarray scanner (PerkinElmer Life and Analytical Sciences Inc., Waltham, MA, USA).

\section{Results}

Prevalence of HSIL and SCC involving ECP. During a study period of 12 years, we identified 1,905 patients with ECP, and most of the them were diagnosed with ECP without additional descriptors. According to the histological criteria for HSIL and SCC $(5,6), 21$ and two patients each had HSIL and SCC involving ECP. The prevalence of HSIL and SCC involving ECP was $1.1 \%(21 / 1,905)$ and $0.1 \%(2 / 1,905)$, respectively.

Clinical characteristics. Table I summarizes the clinicopathological characteristics of the patients with HSIL and SCC involving ECP. The two patients with SCC were aged 53 and 63 (mean: 58) years and were postmenopausal. The mean age of the 21 patients with HSIL was 49.9 (range $=30-84$ ) years and eight $(38.1 \%)$ of these patients were aged $<45$ years and premenopausal.

All except one patient underwent polypectomy under hysteroscopic or colposcopic guidance, which revealed ECP with various gross morphologies (Figure 1). The remaining one patient underwent hysterectomy without polypectomy. Nine $(42.8 \%)$ HSIL patients were treated by cervical conization after polypectomy, and three of those also underwent total hysterectomy following conization. Four $(19.0 \%)$ HSIL patients underwent only hysterectomy. One of the two patients with SCC was treated by radical hysterectomy with bilateral external iliac lymph node sampling, whereas the other patient underwent conization with no further treatment after. None of the patients received postoperative adjuvant chemotherapy or radiation therapy. 
Table II. Pathological characteristics of high-grade squamous intraepithelial lesion (HSIL) and squamous cell carcinoma (SCC) involving endocervical polyp $(E C P)$.

\begin{tabular}{|c|c|c|c|c|}
\hline \multirow[t]{2}{*}{ Characteristic } & & \multicolumn{2}{|c|}{ Polypectomy diagnosis } & \multirow[t]{2}{*}{ Total } \\
\hline & & HSIL & SCC & \\
\hline \multirow[t]{3}{*}{ Greatest size of ECP } & Mean (range) & $13.7(2-40)$ & $15.5(15-16)$ & $13.9(2-40)$ \\
\hline & $<15 \mathrm{~mm}$ & 13 & 0 & 13 \\
\hline & $\geq 15 \mathrm{~mm}$ & 8 & 2 & 10 \\
\hline \multirow[t]{3}{*}{ Greatest size of SCC } & $<3 \mathrm{~mm}$ & Not applicable & $2(<1 \mathrm{~mm})$ & 2 \\
\hline & $<5 \mathrm{~mm}$ & Not applicable & 0 & 0 \\
\hline & $\geq 5 \mathrm{~mm}$ & Not applicable & 0 & 0 \\
\hline \multirow[t]{2}{*}{ Glandular extension } & Present & 19 & 2 & 21 \\
\hline & Absent & 2 & 0 & 2 \\
\hline \multirow[t]{2}{*}{ Bizarre nuclear atypia } & Present & 6 & 1 & 4 \\
\hline & Absent & 15 & 1 & 19 \\
\hline \multirow[t]{2}{*}{ Atypical mitotic figure } & Present & 5 & 2 & 7 \\
\hline & Absent & 16 & 0 & 16 \\
\hline \multirow[t]{3}{*}{ Polypectomy resection margin involvement } & Present & 5 & 1 (by HSIL) & 6 \\
\hline & Absent & 14 & 1 & 15 \\
\hline & Not applicable & 1 & 0 & 1 \\
\hline \multirow[t]{3}{*}{ Conization diagnosis } & Residual SCC & 0 & 0 & 0 \\
\hline & Residual HSIL & 5 & 1 & 6 \\
\hline & No residual lesion & 5 & 0 & 5 \\
\hline \multirow[t]{2}{*}{ Conization resection margin involvement } & Present & 0 & Not applicable & 0 \\
\hline & Absent & 10 & Not applicable & 10 \\
\hline \multirow[t]{3}{*}{ Hysterectomy diagnosis } & Residual SCC & 0 & 0 & 0 \\
\hline & Residual HSIL & 2 & 1 & 3 \\
\hline & No residual lesion & 5 & 0 & 5 \\
\hline
\end{tabular}

Recurrent disease and metastasis did not arise in the two patients with SCC during postoperative follow-up for 11 and 21 months, respectively. The patients with HSIL were followed up for a mean of 44.3 months (range=7-173 months). All $17(81.0 \%)$ patients with HSIL who had complete follow-up information have remained alive without evidence of recurrent disease.

HPV status. Information about HPV genotypes was available for $18(78.3 \%)$ patients: 17 patients with HSIL and one patient with SCC. High-risk HPV was detected in 15 patients with HSIL and in one with SCC (type 16). The most prevalent genotype in the patients with HSIL was type 16 $(7 / 15 ; 46.7 \%)$, followed by types $31(2 / 15 ; 13.3 \%)$ and 52 $(2 / 15 ; 13.3 \%)$. Types $18,33,39$, and 68 were also detected. High-risk HPV was not detected in the remaining two patients with HSIL.

Cytohistological correlation. Cervicovaginal cytology results were available for $19(82.6 \%)$ patients. The findings for two SCC patients were SCC and atypical squamous cells of undetermined significance (ASC-US), respectively. Seventeen patients with HSIL were diagnosed with atypical squamous cells, cannot exclude HSIL (ASC-H; 6/17; 35.3\%),
HSIL (5/17; 29.4\%), ASC-US (4/17; 23.5\%), low-grade squamous intraepithelial lesion (LSIL; 1/17; 5.9\%), and negative for intraepithelial lesion or malignancy (NILM; $1 / 17 ; 5.9 \%)$. Taken together, ASC-H, HSIL, or SCC was diagnosed in $12(63.1 \%)$ of the 19 patients with cytological results.

Pathological characteristics. Table II summarizes pathological characteristics. The mean size of ECP was 13.9 $\mathrm{mm}$ (range $=2-40 \mathrm{~mm}$ ). The mean size of two ECP with SCC was $15.5(15-16) \mathrm{mm}$. Three and 18 patients with HSIL were classified as cervical intraepithelial neoplasia (CIN) 2 and CIN 3, respectively. The mean sizes of the largest ECP classified as CIN 2 and CIN 3 were 5 and $15.2 \mathrm{~mm}$, respectively. Endocervical glandular extension by HSIL was identified in $19(90.5 \%)$ patients with HSIL and two $(100.0 \%)$ with SCC. Bizarre nuclear atypia, including marked nuclear enlargement (4-5-fold larger than basal cell nuclei) and severe pleomorphism, were found in six $(28.6 \%)$ patients with HSIL and one $(50.0 \%)$ with SCC. Mitotic activity was increased in all patients examined. Atypical mitotic figures, including tripolar, tetrapolar, and asymmetric mitoses, were detected in five (23.8\%) and two (100.0\%) patients with HSIL and SCC, respectively. The greatest size 

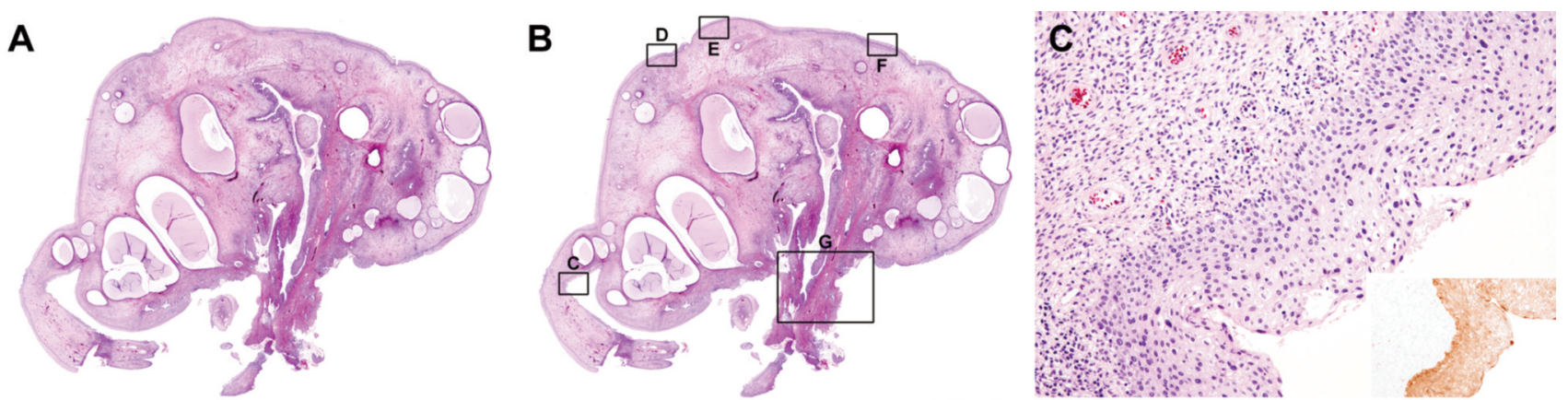

D
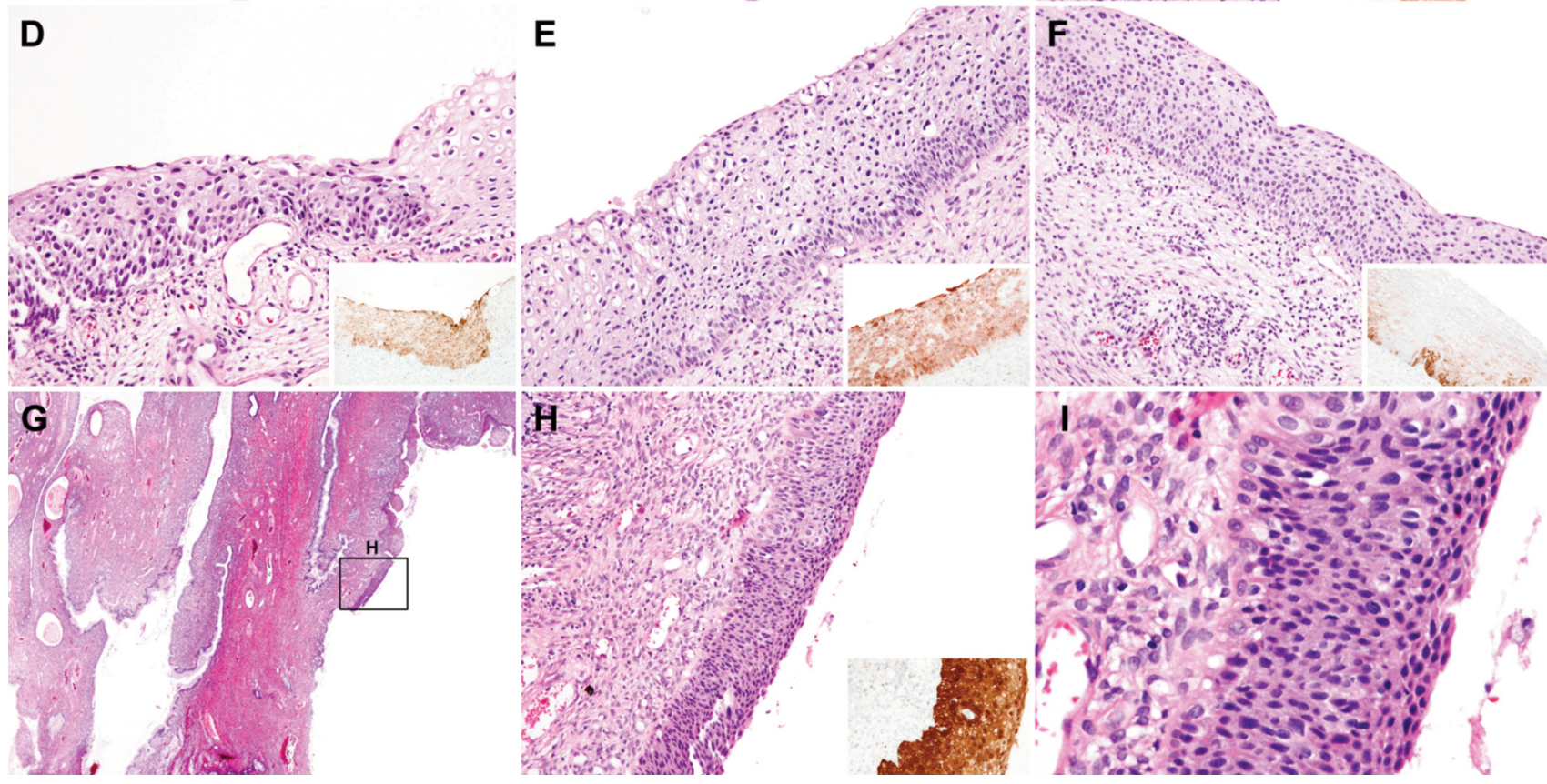

Figure 2. Histopathological features of endocervical polyp (ECP) in case 10. (A) Scan view. (B) Boxes indicate location of subsequent mediumpower views. (C to F) Several areas show atypical squamous lesions along ECP surface and morphologically low-to high-grade squamous intraepithelial lesions, namely suspected cervical intraepithelial neoplasia (CIN) 2. Box (right lower corner) in each image shows p16 patchy positivity, indicating low-grade squamous intraepithelial lesion. (G) Another atypical squamous lesion is located at mid-portion of stalk. (H) Mediumpower view reveals full-thickness proliferation of homogeneous squamous epithelium, consistent with high-grade squamous intraepithelial lesion (HSIL; CIN 3). Box (right lower corner) shows pl6 block positivity. (I). High- power view shows nuclear hyperchromasia and pleomorphism occupying entire epithelial thickness.

and invasion depth of invasive carcinoma were $<1 \mathrm{~mm}$ in both patients with SCC (International Federation of Gynecology and Obstetrics stage IA1). In three representative cases, detailed histopathological features of HSIL and SCC involving ECP are as follows.

Case 10: An umbrella-shaped, pedunculated polyp included several, round-to-ovoid, dilated endocervical glands (Figures 2A, B) and randomly scattered glandular lumina filled with mucin. A few centrally located glands also stretched along the long axis of the polyp. Fibrotic stroma contained some thick-walled blood vessels and ectatic veins. Areas of atypical squamous epithelium were patchily distributed along the surface of the polyp, with variable degrees of nuclear enlargement, hyperchromasia, and pleomorphism. Several foci showing suspected CIN 2 were observed (Figures 2C-F), but p16 immunostaining was patchy positive, which does not support the diagnosis of HSIL. A few microscopic areas of definite HSIL were p16 block positive (Figures 2G-H). The homogeneous proliferation of atypical squamous epithelial cells with severe nuclear pleomorphism at the basal and parabasal layers extending throughout the entire epithelial thickness were characteristic of HSIL (Figure 2I).

Case 11: An ovoid polyp with a short stalk had a central, ovoid, dilated glandular lumen containing clear mucinous material (Figures 3A-B). The surface of the head was almost 

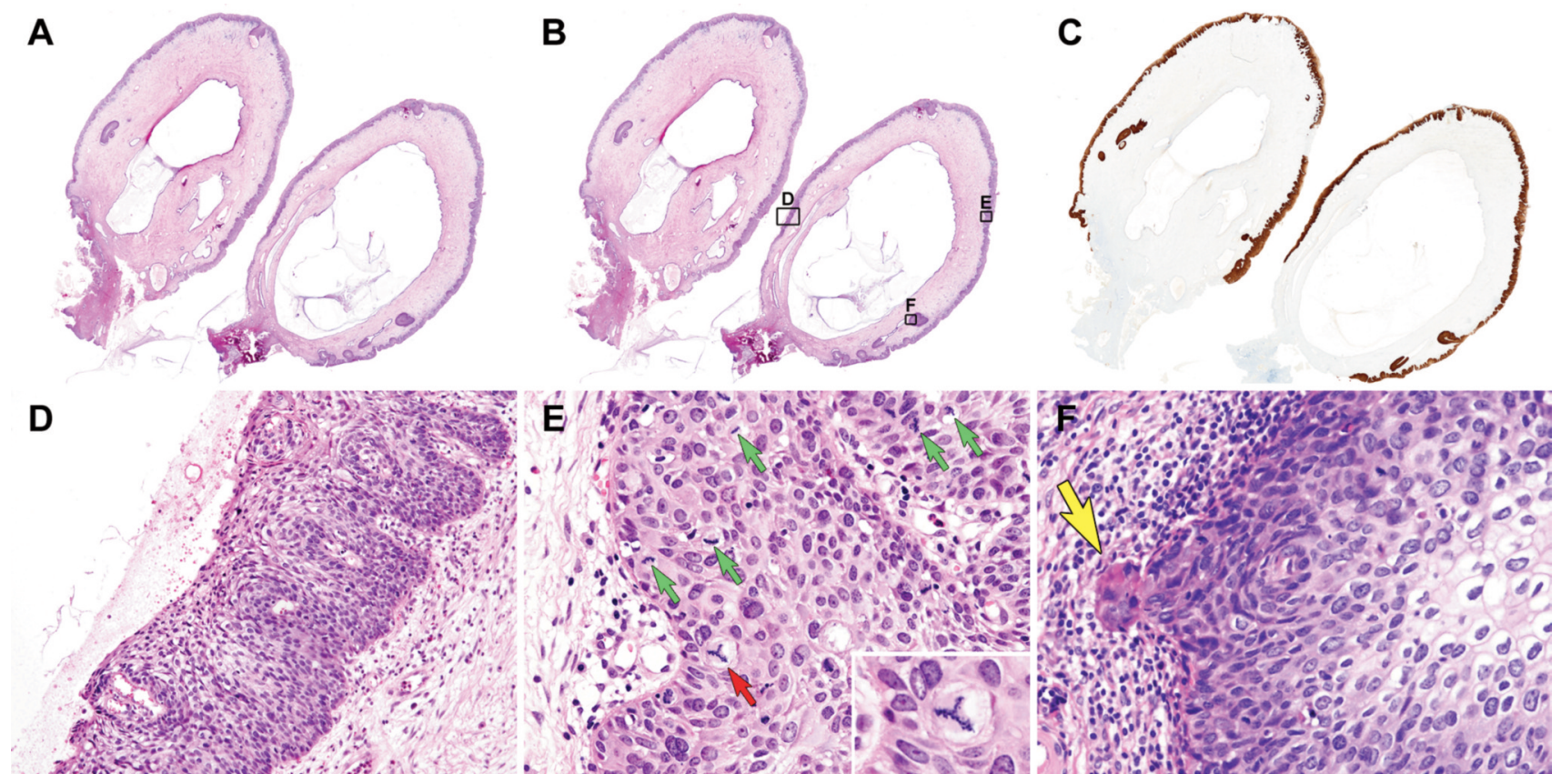

Figure 3. Histopathological features of endocervical polyp: case 11. (A) Scan view. (B) Boxes indicate location of subsequent medium-power views. (C) Immunostaining highlights high-grade squamous intraepithelial lesion (HSIL) with p16 block positivity. Most of polyp surface involves HSIL but not stalk or resection margin. (D) Medium-power view shows HSIL. Mild lymphocytic infiltration is evident in subepithelial stroma. (E) Highpower view shows frequent mitotic figures (small green arrows). Small red arrow shows typical mitotic figure. Box (right lower corner) shows tripolar mitosis. $(F)$ Large yellow arrow indicates microscopic stromal invasion, characterized by protrusion of tumor cells from stromal-epithelial junction into stroma. Invasive focus measures $<1 \mathrm{~mm}$.

totally consumed by HSIL, with p16 block positivity throughout the surface (Figure 3C), and HSIL extensively involved the endocervical glands embedded within the stroma. Nuclear hyperchromasia, pleomorphism (Figure 3D), frequent mitotic figures and a few atypical mitoses were features of tumor cells (Figure 3E). A small microscopic nest of tumor cells in a single focus in the stromal-epithelial junction penetrated the stroma (Figure $3 \mathrm{~F}$ ). The maximum size and invasion depth of SCC were $<1 \mathrm{~mm}$.

Case 12: A leaf-shaped polyp featured a few centrally located, elongated, dilated glandular lumina and a broad base without a stalk (Figures 4A, B). Focal inflammatory infiltrates with granulation tissue at the tip of the polyp were probably due to chronic irritation. The HSIL extensively involved the endocervical glands with expansile growth (Figures 4C-E), and some had a keratinized surface (keratinizing HSIL). Immunostaining revealed p16 block positivity.

The resection margin was involved in polypectomy specimens of five patients with HSIL $(23.8 \%)$ and in one with SCC. One patient with SCC underwent radical hysterectomy. Four HSIL patients received further treatment, and one did not. Two of the four patients with HSIL were treated only by total hysterectomy, and the other two were treated by conization and subsequent total hysterectomy. All the five hysterectomy or conization specimens except from the one patient who was not further treated had residual HSIL. Furthermore, conization specimens revealed residual HSIL in two HSIL patients and in one SCC patient, whose polypectomy resection margins were negative for HSIL or SCC. Chronic cervicitis with reactive epithelial atypia and squamous and tubal metaplasia, hemorrhage, and focal fibrosis were identified in the previous conization bed of conization specimens with no apparent residual lesions. The conization resection margin was not involved in any of these patients.

\section{Discussion}

Berzolla et al. (3) reported that the prevalence of dysplasia and malignancy arising in ECP is $0.5 \%(12 / 2,246)$ and $0.09 \%(2 / 2,246)$, respectively. Similarly, Chin et al. (23) reported that the prevalence rate of SIL involving ECP is $0.4 \%(7 / 1,900)$. In a larger cohort study by Long et al. (24), the overall risk of dysplasia arising in ECP was $0.3 \%$ $(9 / 3,256)$. Here, we analyzed the prevalence of HSIL and SCC in a cohort of Korean patients with ECP. The prevalence of HSIL $(1.1 \% ; 21 / 1,905)$ and $\operatorname{SCC}(0.1 \%$; 


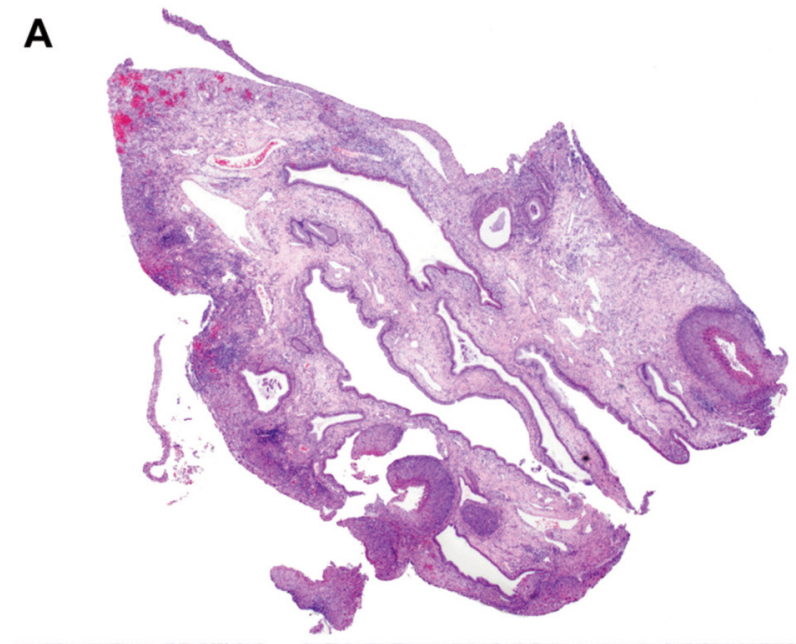

B
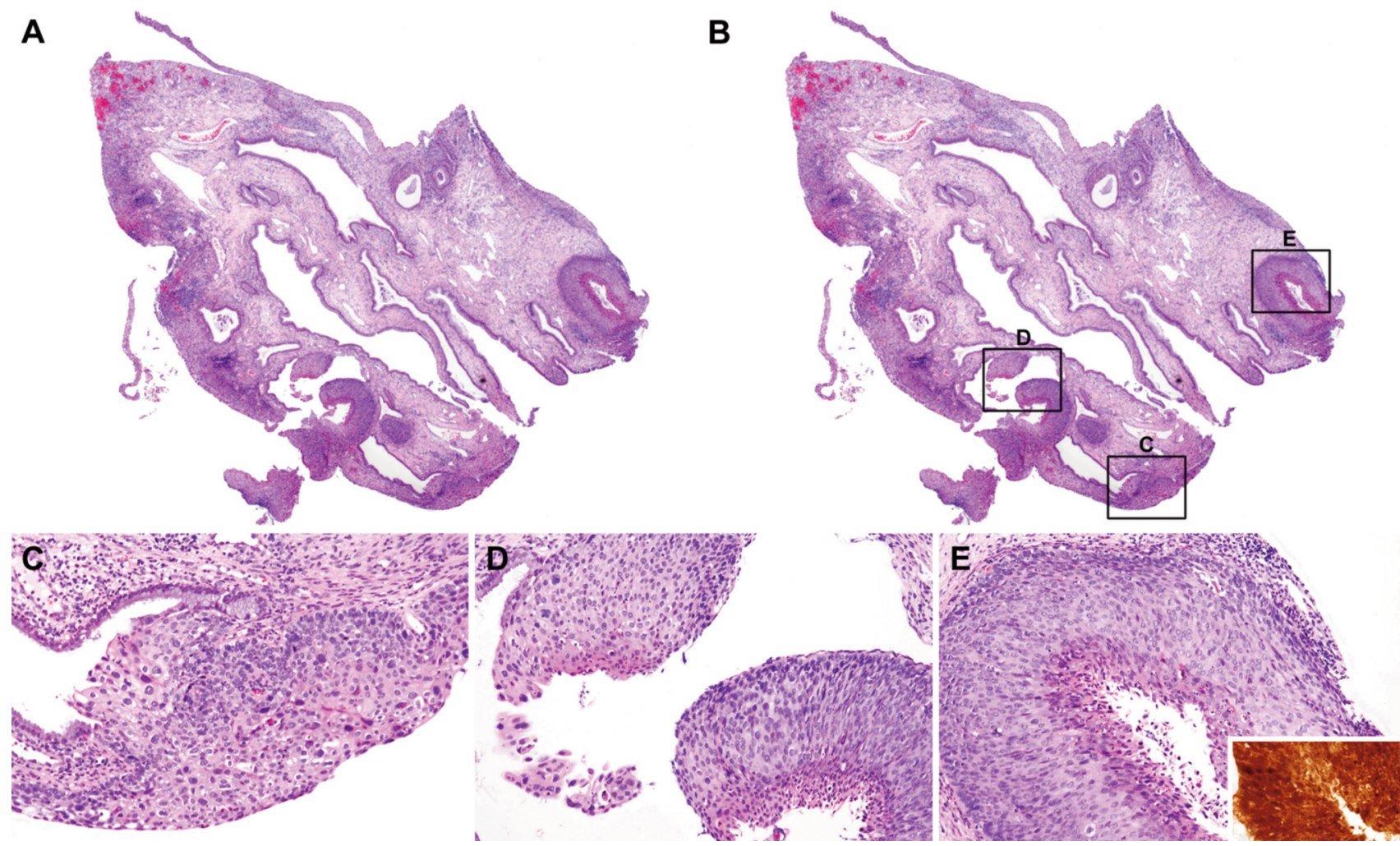

Figure 4. Histopathological features of endocervical polyp: case 12. (A) Scan view. (B) Boxes indicate location of subsequent medium-power views. (C to E) Extensive, multifocal glandular extension of high-grade squamous intraepithelial lesion (HSIL). Box in right lower corner of image $E$ shows block p16 positivity.

$2 / 1,905)$ involving ECP in our cohort was similar to the rates found in these studies.

Although eight $(38.1 \%)$ of 21 patients with HSIL were aged $<45$ years, the average age of two patients with SCC was 58 years. The younger patients tended to have HSIL than SCC involving ECP, reflecting the fact that HSIL occurs in younger persons and progresses to SCC. Moreover, the number of patients with SCC in the present study was too small to detect a significant difference.

Histological features of invasive carcinoma in two patients with SCC comprised HSIL with protruding microscopic tumor cell nests $<1 \mathrm{~mm}$ that extended from the stromalepithelial junction to the subepithelial stroma, which was unlikely to be visualized by the naked eye. Moreover, the HSIL distribution on polyp surfaces was random and in various sizes; thus, identifying uninvolved regions with the naked eye was difficult. The gross appearance of the polyp surface varied regardless of HSIL as a result of erosion, inflammation, granulation tissue formation, and fibrosis from chronic irritation.

The rate of concordance between the presence of HSIL or SCC and high-risk HPV detection in the present study was $88.2 \%$ (16/18). Furthermore, when considering ASC-H and worse as a standard for cytological interpretation, the cytohistological concordance rate was $63.1 \%$ (12/19). The reason for this was most likely sampling error due to ECP being located within the endocervix and not being found during routine speculum or colposcopic examination. We also found that patients with discordant results had noncontinuous, randomly distributed HSIL areas admixed with squamous metaplasia and multifocal LSIL. In contrast, most patients with concordant results had continuous HSIL that involved most of the polyp surface and many had exposure outside the external os due to large polyps. Taken together, our results suggest that when HSIL or SCC involves ECP, the diagnostic value derived from HPV tests or cytological examinations would be limited.

Our results indicate that there are limitations in terms of predicting concurrent HSIL or SCC from colposcopic appearance, HPV status, or cytological results prior to polypectomy. Therefore, a thorough pathological examination after polypectomy is warranted so that clinicians can be informed about resection margin involvement. Five of 23 of our patients had HSIL involving the polypectomy resection margin. One patient with SCC underwent radical hysterectomy, and two patients each with HSIL underwent 
total hysterectomy and conization. Tissues removed from all five patients showed residual HSIL. In the absence of clear guidelines for the method of polypectomy or changing its regions, these results suggest that involvement of the polypectomy resection margin is an important factor in predicting residual HSIL even when HSIL or SCC involving ECP is not clinically suspected. Hysterectomy will be needed for patients with polypectomy resection margin involvement to remove possible residual lesions, and therapeutic conization will be necessary for those whose fertility should be maintained. However, polypectomy resection margin involvement cannot be concluded as an absolute predictor marker for residual HSIL. This is because residual HSIL was found in two patients with HSIL who did not have polypectomy resection margin involvement and in conization specimens from one patient with SCC. These findings suggest that a separate HSIL can exist in nonpolypoid cervical mucosa, and that additional treatment will be necessary regardless of polypectomy resection margin involvement.

When HSIL was accompanied by ECP, the mean size of the largest polyp with histological grade CIN 2 was smaller than that of CIN 3 (5 vs. $15.2 \mathrm{~mm}$ ). However, the significance remains indeterminate, as only three patients had CIN 2. Differences in the mean largest size of ECP (13.9 vs. $15.5 \mathrm{~mm}$ ) in HSIL and SCC could not be statistically compared, because only two patients had SCC. The rate and extent of the unusual histological features of HSIL, such as marked nuclear enlargement, severe nuclear pleomorphism, and atypical mitotic figures, did not significantly differ between patients with HSIL and SCC. This finding is in agreement with that of a study by Stewart (25) who reported that pleomorphic epithelial changes (marked nuclear atypia often associated with multinucleation) in HSIL do not necessarily indicate more aggressive biological behavior.

None of the patients, including the eight who did not undergo further treatment, had evidence of recurrence or metastasis during follow-up after polypectomy. Of those who did not undergo further treatment, two patients were lost to follow-up and four were followed up for less than 3 years. Therefore, observation after polypectomy should not be mistaken as a treatment option. Patients diagnosed with HSIL and SCC involving ECP should undergo treatment according to the management guidelines for HSIL and SCC without ECP until more clinical data are collected to determine the behavior. Patients in the present study were followed up for a relatively short period. Therefore, future studies should analyze the clinical course and outcomes of HSIL and SCC involving ECP in a larger patient cohort over a longer follow-up period.

In conclusion, the prevalence of HSIL (1.1\%) and SCC $(0.1 \%)$ involving ECP in our cohort was similar to that is previous studies. The presence of residual HSIL in nonpolypoid cervical tissues in cases with polypectomy resection margin involvement suggest that conization or hysterectomy is needed for diagnostic or treatment purposes. Since cervicovaginal cytology or HPV tests have insufficient diagnostic value as a screening tool for HSIL and SCC involving ECP, a thorough pathological examination of polypectomy specimens is required to provide sufficient clinicopathological information to clinicians for appropriate management.

\section{Conflicts of Interest}

The Authors declare that they have no conflicts of interest.

\section{Authors' Contributions}

All Authors made substantial contributions to the conception and design of the study; the acquisition, analysis, and interpretation of the data; drafting of the article; critical revision of the article for important intellectual content; and the final approval of the version to be published.

\section{Acknowledgements}

This research was supported by the Chungnam National University Hospital Research Fund (2019), by the National Research Foundation of Korea (NRF) grant funded by the Korea government (Ministry of Science and ICT) (2019R1G1A1100578), and by a Grant of Translational R\&D Project through Institute for BioMedical Convergence (Incheon St. Mary's Hospital, College of Medicine, The Catholic University of Korea).

\section{References}

1 Farrar HK, Jr. and Nedoss BR: Benign tumors of the uterine cervix. Am J Obstet Gynecol 81: 124-137, 1961. PMID: 13698265. DOI: $10.1016 /$ s0002-9378(16)36314-1

2 Schnatz PF, Ricci S and O'Sullivan DM: Cervical polyps in postmenopausal women: is there a difference in risk? Menopause 16: 524-528, 2009. PMID: 19179926. DOI: $10.1097 /$ gme.0b013e3181927286

3 Berzolla CE, Schnatz PF, O’Sullivan DM, Bansal R, Mandavilli $\mathrm{S}$ and Sorosky JI: Dysplasia and malignancy in endocervical polyps. J Womens Health 16: 1317-1321, 2007. PMID: 18001188. DOI: $10.1089 /$ jwh.2007.0408

4 Golan A, Ber A, Wolman I and David MP: Cervical polyp: evaluation of current treatment. Gynecol Obstet Invest 37: 5658, 1994. PMID: 8125411.

5 Kurman RJ, Carcangiu ML, Herrington CS and Young RH: WHO Classification of Tumours of Female Reproductive Organs. IARC: Lyon, France, 2014.

6 Darragh TM, Colgan TJ, Thomas Cox J, Heller DS, Henry MR, Luff RD, McCalmont T, Nayar R, Palefsky JM, Stoler MH, Wilkinson EJ, Zaino RJ, Wilbur DC and Members of the LPWG: The Lower Anogenital Squamous Terminology Standardization project for HPV-associated lesions: background and consensus recommendations from the College of American Pathologists and the American Society for Colposcopy and Cervical Pathology. Int J Gynecol Pathol 32: 76-115, 2013. PMID: 23202792. DOI: $10.1097 /$ PGP.0b013e31826916c7 
7 Bae GE, Do SI, Kim K, Park JH, Cho S and Kim HS: Increased sphingosine kinase 1 expression predicts distant metastasis and poor outcome in patients with colorectal cancer. Anticancer Res 39: 663-670, 2019. PMID: 30711943. DOI: 10.21873/anticanres. 13161

8 Bae GE, Yoon N, Cho EY, Kim HS and Cho SY: Clinicopathological and molecular characteristics of mammary adenoid cystic carcinoma with adipocytic differentiation with emphasis on the identification of a novel BRAF mutation. Anticancer Res 39: 369-374, 2019. PMID: 30591482. DOI: 10.21873/anticanres.13121

9 Chung T, Do SI, Na K, Kim G, Jeong YI, Kim YW and Kim HS: Stromal p16 overexpression in gastric-type mucinous carcinoma of the uterine cervix. Anticancer Res 38: 3551-3558, 2018. PMID: 29848709. DOI: 10.21873/anticanres.12627

10 Joo JW, Kim HS, Do SI and Sung JY: Expression of zinc finger and BTB domain-containing 7A in colorectal carcinoma. Anticancer Res 38: 2787-2792, 2018. PMID: 29715100. DOI: 10.21873/anticanres. 12522

11 Jung YY, Sung JY, Kim JY and Kim HS: Down-regulation of Bcell translocation gene 1 by promoter methylation in colorectal carcinoma. Anticancer Res 38: 691-697, 2018. PMID: 29374692. DOI: 10.21873 /anticanres.12274

12 Park CK and Kim HS: Clinicopathological characteristics of ovarian metastasis from colorectal and pancreatobiliary carcinomas mimicking primary ovarian mucinous tumor. Anticancer Res 38: 5465-5473, 2018. PMID: 30194204. DOI: 10.21873/anticanres.12879

13 Sung JY, Jung YY and Kim HS: Clinicopathological characteristics and KRAS mutation status of endometrial mucinous metaplasia and carcinoma. Anticancer Res 38: 27792786, 2018. PMID: 29715099. DOI: 10.21873/anticanres.12521

$14 \mathrm{Na} \mathrm{K}$ and Kim HS: Clinicopathologic and molecular characteristics of mesonephric adenocarcinoma arising from the uterine body. Am J Surg Pathol 43: 12-25, 2019. PMID: 29189288. DOI: 10.1097/PAS.0000000000000991

15 Jang MI, Sung JY, Kim JY and Kim HS: Clinicopathological characteristics of metaplastic papillary tumor of the fallopian tube. Anticancer Res 37: 3693-3701, 2017. PMID: 28668862.

$16 \mathrm{Na} \mathrm{K}$ and Kim HS: Clinicopathological characteristics of fallopian tube metastases from primary endometrial, cervical, and nongynecological malignancies: A single institutional experience. Virchows Arch 471: 363-373, 2017. PMID: 28702779. DOI: $10.1007 / \mathrm{s} 00428-017-2186-\mathrm{Z}$

$17 \mathrm{Na} \mathrm{K}$, Lee JY, Sung JY, Kim GM, Koo JS and Kim HS: Comparative clinicopathological and cytomorphological analyses of peritoneal carcinomatosis associated with metastatic breast carcinoma and primary peritoneal/ovarian carcinoma in patients with a history of breast carcinoma. Virchows Arch 473: 165-175, 2018. PMID: 29926183. DOI: 10.1007/s00428-018-2390-5
18 Na K, Sung JY and Kim HS: Stromal p16 overexpression in adult granulosa cell tumors of the ovary. Anticancer Res 37: 2437-2444, 2017. PMID: 28476811. DOI: 10.21873/anticanres. 11583

19 Na K, Sung JY and Kim HS: TP53 mutation status of tuboovarian and peritoneal high-grade serous carcinoma with a wildtype p53 immunostaining pattern. Anticancer Res 37: $6697-$ 6703, 2017. PMID: 29187446. DOI: 10.21873/anticanres.12128

20 Bae GE, Yoon G, Song YJ and Kim HS: High-grade squamous intraepithelial lesion arising adjacent to vulvar lymphangioma circumscriptum: a tertiary institutional experience. Oncotarget 7: 48120-48129, 2016. PMID: 27329721. DOI: 10.18632/ oncotarget. 10158

21 Jung YY, Nahm JH and Kim HS: Cytomorphological characteristics of glassy cell carcinoma of the uterine cervix: histopathological correlation and human papillomavirus genotyping. Oncotarget 7: 74152-74161, 2016. PMID: 27708230. DOI: 10.18632/oncotarget.12361

22 Yoon N, Kim JY and Kim HS: Clinical outcomes of advancedstage glassy cell carcinoma of the uterine cervix: a need for reappraisal. Oncotarget 7: 78448-78454, 2016. PMID: 27793022. DOI: $10.18632 /$ oncotarget.12905

23 Chin N, Platt AB and Nuovo GJ: Squamous intraepithelial lesions arising in benign endocervical polyps: a report of 9 cases with correlation to the Pap smears, HPV analysis, and immunoprofile. Int J Gynecol Pathol 27: 582-590, 2008. PMID: 18753960. DOI: 10.1097/PGP.0b013e31817e0928

24 Long ME, Dwarica DS, Kastner TM, Gallenberg MM, Chantigian PD, Marnach ML, Weaver AL and Casey PM: Comparison of dysplastic and benign endocervical polyps. J Low Genit Tract Dis 17: 142-146, 2013. PMID: 22885648. DOI: 10.1097/LGT.0b013e318260e32f

25 Stewart CJR: High-grade squamous intraepithelial lesion (HSIL) of the cervix with bizarre cytological appearances ('pleomorphic HSIL'): a review of 19 cases. Pathology 49: 465-470, 2017. PMID: 28666641. DOI: 10.1016/j.pathol.2017.05.002
Received June 2, 2020

Revised June 22, 2020

Accepted June 24, 2020 